\title{
Intracluster Planetary Nebulae in Virgo: Photometric selection, spectroscopic validation and cluster depth ${ }^{1}$
}

\author{
Magda Arnaboldi \\ Astronomical Observatory of Capodimonte, Via Moiariello 16, I-80131 Naples \\ magda@na.astro.it \\ J. Alfonso L. Aguerri \\ Astronomisches Institut der Universität Basel, Venusstrasse 7, Binningen, Switzerland \\ jalfonso@astro.unibas.ch \\ Nicola R. Napolitano \\ Astronomical Observatory of Capodimonte, Via Moiariello 16, I-80131 Naples \\ Dept. of Physics, University "Federico II", I-80125 Naples \\ napolita@na.astro.it \\ Ortwin Gerhard \\ Astronomisches Institut der Universität Basel, Venusstrasse 7, Binningen, Switzerland \\ gerhard@astro.unibas.ch \\ Kenneth C. Freeman \\ RSAA, Mt. Stromlo Observatory, Weston Creek P.O., ACT 2611 \\ kcf@mso.anu .edu.au \\ John Feldmeier \\ Case Western Reserve University, 10900 Euclid Avenue, Cleveland, OH 44106-7215 \\ johnf@eor . astr. cwru.edu \\ Massimo Capaccioli \\ Astronomical Observatory of Capodimonte, Via Moiariello 16, I-80131 Naples \\ Dept. of Physics, University "Federico II", I-80125 Naples \\ capaccioli@na.astro.it \\ Rolf P. Kudritzki
}


Institute for Astronomy, Honolulu, Hawaii 96822 USA

kud@ifa.hawaii.edu

Roberto H. Méndez

Munich University Observatory, Scheinerstr. 1, D-81679 Munich

mendez@usm.uni-muenchen.de

\begin{abstract}
We have imaged an empty area of $34 \times 34 \operatorname{arcmin}^{2}$ one and a half degree north of the Virgo cluster core to survey for intracluster planetary nebula candidates. We have implemented and tested a fully automatic procedure for the selection of emission line objects in wide-field images, based on the on-off technique from Ciardullo and Jacoby. Freeman et al. have spectroscopically confirmed a sample of intracluster planetary nebulae in one Virgo field. We use the photometric and morphological properties of this sample to test our selection procedure. In our newly surveyed Virgo field, 75 objects were identified as best candidates for intracluster PNe.

The luminosity function of the spectroscopically confirmed PNe shows a brighter cutoff than the planetary nebula luminosity function for the inner regions of M87. Such a brighter cut-off is also observed in the newly surveyed field and indicates a smaller distance modulus, implying that the front end of the Virgo cluster is closer to us by a significant amount: $14 \%$ closer $(2.1 \mathrm{Mpc})$ than M87 for the spectroscopic field, using the PN luminosity function distance of $14.9 \mathrm{Mpc}$ to M87, and $19 \%$ closer (2.8 Mpc) than M87 for the newly surveyed field. Independent distance indicators (Tully-Fisher relation for Virgo spirals and surface brightness fluctuations for Virgo ellipticals) agree with these findings.

From these two Virgo cluster fields there is no evidence that the surface luminosity density for the diffuse stellar component in the cluster decreases with radius. The luminosity surface density of the diffuse stellar population is comparable to that of the galaxies.
\end{abstract}

Subject headings: clusters: galaxies, dynamics, planetary nebulae

\footnotetext{
${ }^{1}$ This paper is based on observations carried out at the ESO telescopes at La Silla and at the Anglo Australian Observatory.
} 


\section{Introduction}

The presence of a diffuse intracluster light filling the regions between galaxies in clusters has been known for several decades. In his introduction to the study of the galaxy luminosity function in the Coma cluster in 1951, Zwicky suggested that “... Vast and often very irregular swarms of stars and other matter exist in the spaces between the conventional spiral, elliptical, and irregular galaxies. It is not at all clear how such swarms should be incorporated into any distribution function of galaxies.." (Zwicky 1951). Early studies of the diffuse light in clusters were based on photographic techniques (Welch \& Sastry 1971; Melnick et al. 1977; Thuan \& Kormendy 1977), and were followed in the 1990s by CCD observations (Guldheus 1989; Uson et al. 1991; Bernstein et al. 1995; Vilchez-Gómez et al. 1994). The limitations of photographic photometry and the small CCD fields used in these works lead to large uncertainties in the results. The best estimates for the contribution of the diffuse light ranged from $10 \%$ to $50 \%$ of the total light emitted by cluster galaxies in the central regions of the clusters. Such studies, which need accurate photometry on large sky areas, encountered two problems: 1) the typical surface brightness of the intracluster light is less than $1 \%$ of the sky brightness and 2) it is difficult to distinguish between the diffuse light associated with the halo of the cD galaxy and that associated with the cluster (Bernstein et al. 1995).

A different method for probing intracluster light is through the direct detection of the stars themselves. In 1977 a search was done for intergalactic supernovae in the Coma cluster (Crane et al. 1977), but all the supernovae detected were found to be associated with Coma galaxies. In 1981 a supernova Type Ia was discovered in the region between M86 and M84 in the Virgo cluster (Smith 1981).

A new approach came from the discovery of intracluster planetary nebulae (ICPNe) in the Virgo and Fornax clusters. In a radial velocity survey of planetary nebulae (PNe) in NGC $4406\left(\mathrm{v}_{\text {sys }}=-227 \mathrm{kms}^{-1}\right)$, three objects were found with radial velocities $v>1300 \mathrm{kms}^{-1}$ (Arnaboldi et al. 1996). Theuns \& Warren (1997) found 10 ICPNe candidates in the Fornax cluster and Ferguson et al. (1998) used HST to detect a statistical excess of red stars in a field near M87 relative to the Hubble Deep Field; for follow-up studies of intracluster light in Virgo see also Durrell et al. (2001). Further surveys in Virgo (Méndez et al. 1997; Ciardullo et al. 1998; Feldmeier et al. 1998; Feldmeier 2000) in the [OIII] $\lambda 5007$ line resulted in the identification of more than a hundred ICPNe candidates in the Virgo core region.

The first spectroscopic follow-up (Kudritzki et al. 2000; Freeman et al. 2000) carried out on subsamples of these catalogues showed that the on-off technique plus the "by-eye" identification of the PN candidates included some stars which were mis-classified as emission line objects. In addition, because of the very faint limiting fluxes reached by these surveys, they also detect high redshift emission-line objects, most probably from Ly $\alpha$ emitters at redshift 3.13 and [OII] emitters at redshift 0.347 .

We have started a survey project which aims at detecting ICPNe in large areas in the Virgo cluster and tracing their number density profile from the Virgo cluster center. The large CCD

mosaic images used in this project require the implementation of a robust automatic procedure to 
reliably select a population of emission line candidates. In this paper we present the survey carried out in one field at an angular distance of $1.5 \mathrm{deg}$ from the Virgo core (Binggeli et al. 1987), and discuss in some detail the selection criteria adopted for the identification of ICPNe. A critical step in this procedure is the validation against a spectroscopically confirmed sample of ICPNe obtained by Freeeman et al. (2000, 2001 in prep.).

The survey of ICPNe in Virgo associated with the diffuse stellar population is particularly relevant in the ongoing discussion for the 3D shape of the Virgo cluster. The planetary nebulae luminosity function (PNLF) has been extensively used as distance indicator for early-type galaxies in Virgo and Fornax (Jacoby et al. 1990; McMillan et al. 1993; Méndez et al. 2001). Therefore the ICPNe luminosity function can be a useful trace of the Virgo cluster structure. Other distance indicators have advocated a significant depth for the Virgo cluster. The Tully-Fisher relation (Pierce \& Tully 1988; Tonry et al. 1990; Yasuda et al. 1997) supports a distance range of 12 to $20 \mathrm{Mpc}$ for the Virgo spirals. Surface brightness fluctuations for early-type galaxies (Neilsen \& Tsvetanov 2000; West \& Blakeslee 2000) place most of the elliptical galaxies at $\sim \pm 2$ Mpc around M87, but indicate a significant fraction of background and foreground objects. Surveys of ICPNe covering large areas in the Virgo cluster would detect preferentially those ICPNe at the closer edge of the Virgo cluster, and therefore trace its extension quite efficiently.

In the following sections, we will review and discuss previous procedures implemented for the selection of PNe in galaxies and empty fields and test a method for the automatic selection of a reliable ICPNe catalogue. In Section 2 we present the new observation of a Virgo cluster field covering $34^{\prime} \times 34^{\prime}$ and the data reduction of the mosaiced data. In Section 3 we present the extraction procedure and test its completeness. In Section 4 we discuss the candidate selection from the extracted catalogue based on their morphology and colors and test them against the spectroscopic validations. In Section 5 we discussed the properties, the luminosity function and the inferred luminosity density in the different fields. Conclusions are given in Section 6.

\section{Imaging observations and data processing}

\subsection{Observations}

In March 1999 we observed a field in the Virgo cluster 1.5 degrees north of the cluster core (Binggeli et al. 1987) at coordinates $\alpha(J 2000)=12: 26: 12.8$ and $\delta(J 2000)=+14: 08: 02.7$; we will refer to this field as the RCN1 field. This field was selected in a region without bright galaxies, to target the intracluster population. We used the WFI on the ESO 2.2-m telescope to image this field through an $80 \AA$ wide filter centred at $5023 \AA$, which is the wavelength of the [OIII] $\lambda 5007 \AA$ emission at the redshift of Virgo cluster (the "on-band" filter), plus a broad V band (the "off-band" filter). The total exposure times were 24000s in the narrow band filter, and 2400s in the V band. Individual frame exposure times were 3000s for the narrow band filter and 300s for the V broad band, and were acquired following an elongated rhombi-like dithering pattern in order to remove 
the CCD gaps, bad pixels and hot/cold columns, plus optimum flat fielding. Both sets of images were taken under similar seeing conditions $\left(\approx 1 \cdot 2^{\prime \prime}\right)$.

Our new field covers a much larger area than previous observations for the detection of ICPNe in Virgo fields (Feldmeier et al. 1998; Méndez et al. 1997; Arnaboldi et al. 1996). The whole image consists of a mosaic of $4 \times 2$ CCD detectors with narrow inter-chip gaps. One pointing of the WFI covers $34^{\prime} \times 34^{\prime}$. The WFI combines a large field of view with a small pixel size of $0.238^{\prime \prime}$. The CCDs have an average read out noise of $4.5 \mathrm{ADU} /$ pixel and a gain of $2.2 \mathrm{e}^{-} / \mathrm{ADU}$. During the night, several Landolt fields and spectrophotometric standard stars were acquired for the broad band and narrow band flux calibrations.

\subsection{Data reduction}

Here we summarise the basic steps for the data reduction: for a detailed treatment of the mosaic data we refer the reader to Capaccioli et al. (2001). The removal of instrumental signature (bias subtraction and flat-field corrections) was performed using the tasks in the MSCRED package in IRAF. The electronic bias level was removed from each CCD by fitting a Chebyshev function to the overscan region and subtracting it from each column. By averaging 10 bias frames, a master bias was created and subtracted from the images in order to remove any remaining bias structures. The dark signal of each CCD detector was also subtracted from the images with the longest exposure time. Two types of flat-fields were taken: twilight and dome flats. Twilight flats were used for removing the large scale structure and the dome flats for the residual pixel-to-pixel structure.

Some structures were still present in the sky background at the edges of the field, even after the twilight flat correction: this was additionally removed using a dark-sky super-flat image. In CCD mosaiced data, superflats are needed because the optics of the wide field imager diffuse light in a different way in twilight flats than in dark-sky images. The superflat was constructed as the median of the un-registered reduced dark-sky science images for each filter independently, with a $3 \times \sigma$ rejection algorithm. The residual structures in the background of the scientific frames are corrected when this superflat is used ${ }^{2}$.

The final images are produced by stacking the dithered set of exposures obtained for each filter. Each reduced exposure must be corrected for the geometric distortions, registered onto a common spatial reference, sky-subtracted and transparency-corrected before the final image can be produced. For further details, see the MSCRED package in IRAF and Capaccioli et al. (2001).

\footnotetext{
${ }^{2}$ Because of the large dithering pattern adopted during image acquisition, any diffuse light structures like ripples, shells or tidal tails as in Calcáneo-Rodán et al. (2000) would be averaged out in the superflat.
} 


\subsection{Flux calibrations}

The strongest emission of a planetary nebula (PN) is frequently the [OIII] $\lambda 5007 \AA$ line (Dopita et al. 1992). The integrated flux from the [OIII] line of a PN is usually expressed in a "m(5007)" magnitude using the definition first introduced by Jacoby (1989):

$$
m(5007)=-2.5 \log F_{5007}-13.74
$$

which gives the PN luminosity in equivalent $\mathrm{V}$ magnitude ${ }^{3}$ and $F_{5007}$ is the total [OIII] $\lambda 5007$ flux in erg $\mathrm{cm}^{-2} \mathrm{~s}^{-1}$ of each PN. We have adopted a different system for the narrow band and broad band fluxes. To compare the limiting fluxes in the on-band and off-band, we normalise fluxes to AB magnitudes, following Theuns \& Warren (1997).

The AB magnitude system is normalised to the Vega flux as follows (Oke 1990):

$$
m_{A B}=-2.5\left(\log F_{\nu}+19.436\right)
$$

The zero points for the narrow and broad band filters in the AB magnitude system were set from observations of the spectroscopic standard stars LTT 7379 and Hilt600. These two stars were offset to each CCD of the mosaic, and the errors on the zero points were determined from the RMS of the zero points from the $8 \mathrm{CCD}$ of the mosaic. Several Landolt fields were observed for the calibration of the $\mathrm{V}$ band image. The zero points for the $[\mathrm{OIII}]$ and $\mathrm{V}$ band imaging in $\mathrm{AB}$ magnitude are $Z_{[O I I I]}=21.08 \pm 0.08$ and $Z_{V}=23.76 \pm 0.08$, normalised to one second exposure. The error of 0.08 on the zero point is dominated by a non-uniform illumination of the WFI mosaic (see Capaccioli et al. 2001 for a detailed discussion of this problem). Airmasses were 1.3 and 1.2 for the reference [OIII] and the $\mathrm{V}$ band exposure ${ }^{4}$ respectively, and we have adopted the average extinction coefficient for the La Silla observatory of $X_{[O I I I]}=0.16$ at $\lambda=5030 \AA$ and of $X_{V}=0.13$ for the $\mathrm{V}$ band.

We have computed the relation between the $m(5007)$ and the $m_{A B}$ magnitudes for the narrow band adopted filter for which

$$
m(5007)=m_{A B}+2.51
$$

additional details on this calibration are found in Appendix A. By using the AB magnitude system, the "on-off" band techniques can be translated to a color selection criterium for an automatically generated catalogue. Aperture magnitudes are computed in a fixed aperture of $3^{\prime \prime}$ diameter (see discussion Sect. 3.1) for all objects, while the Kron magnitudes (mag(auto) in SExtractor) are computed in an aperture with radius equal to $2.5 \times$ the Kron radius (Kron 1980), which scales with the FWHM of the object luminosity profile. The catalogues extracted in our field provide both

\footnotetext{
${ }^{3}$ This definition approximates the apparent $\mathrm{V}$ magnitude one would see if all the [OIII] emission were distributed over the $\mathrm{V}$ bandpass.

${ }^{4}$ The reference $[\mathrm{OIII}]$ and $\mathrm{V}$ band exposures are those exposures in the dithered set of images which had the lowest airmass and best seeing.
} 
aperture and Kron magnitudes for the [OIII] and V band images; the correction between aperture and Kron magnitude is 0.1 in both bands for stellar sources.

\section{Extraction of emission line candidates}

Because of the strong [OIII] $\lambda 5007 \AA$ emission from PNe, they have so far been easily identified via the so called "on-off" band technique: if the field of interest is imaged through a narrow band filter centred at the redshifted [OIII] emission of a PN, and a second image is acquired using an offband filter, PNe candidates are usually identified as those point-like objects which are seen in the on-band image, and not seen in the off-band, when the two images are blinked. This "on-off" band technique and the "by-eye" identification of suitable candidates were used for PNe identifications in Virgo and Fornax ellipticals (Jacoby et al. 1990; McMillan et al. 1993; Ciardullo et al. 1998) and in empty intracluster fields (Méndez et al. 1997; Feldmeier et al. 1998). However, spectroscopic follow-up of ICPNe candidates from (Feldmeier et al. 1998) has shown some contaminations from stars (Kudritzki et al. 2000), which may be caused by an off band image which is not "deep" enough. A more quantitative color selection with an analysis of the limiting magnitudes in each of the two bands will allow a more detailed analysis of the completeness and contamination of the survey.

A survey of large fields requires an automatic procedure for the candidate identification: wide fields and high angular resolution, with image frames of about 64 million pixels, makes the "by-eye" identification on blinking mosaic images difficult and time consuming. The goal of this Section is to implement an automatic detection algorithm, identify a series of optimal selection criteria and apply such a procedure to our newly surveyed RCN1 field. In the next Section, the procedure will be tested against a spectroscopic confirmed ICPNe sample from Freeman et al. (2000, 2001 in prep).

The approach by Theuns \& Warren (1997) is readily automated for large format CCD images. They selected IPNe candidates by their color excess in $m_{n}-m_{v}$ color, where $m_{n}$ is the magnitude of the object in the narrow band filter centred on the line emission, and $m_{v}$ is the $\mathrm{V}$ band, both on in the $\mathrm{AB}$ magnitude system ${ }^{5}$. Their best candidates are those with a significant color excess in the narrow-band filter, i.e. $m_{n}-m_{v}<<0$. This approach can be tested against spectroscopically validated samples.

\footnotetext{
${ }^{5}$ Our off-band filter includes the [OIII] line in its band pass, but we can neglect this contribution because it would be 1.5 magnitudes below our $\mathrm{V}$ band detection limit even for the brighter objects
} 


\subsection{Automatic candidate detections}

We used SExtractor (Bertin \& Arnouts 1996) for the photometry in the broad V and narrow bands. SExtractor identifies and measures fluxes from point-like and extended objects in large format astronomical images. Here we discuss those parameters in SExtractor that are relevant for the identification and flux measurements of our objects; for a detailed description of the software we refer to Bertin \& Arnouts (1996).

Given a set of parameters for the source detection, we perform a set of tests to determine 1) the completeness and 2) the fraction of spurious objects. The parameter set for the source extraction will be the one for which the best compromise will be achieved. We use a catalogue of simulated objects, homogeneously distributed in our field with a given luminosity function, add these simulated objects to the narrow band image, and then look for the best parameter set for the source detection which allows to reach the faintest flux for the modelled objects. The fraction of spurious detections is derived from a simulated image which includes a realistic background plus the modelled catalogue ${ }^{6}$. An optimal parameter set for a source detection with SExtractor is the one which detects the largest number of real objects and produces the smallest fraction of spurious detections, in the last magnitude bin. The fraction of spurious detections depends strongly on the threshold set for source detection and the image noise structure.

The simulated catalogue is computed from a luminosity function (LF) which well reproduces the PNLF for the sample in M31, see Eq. (5), in the [OIII] AB magnitude interval $(22 \div 26)$. We tried detection thresholds between $0.7 \sigma$ and $1.2 \sigma$, where $\sigma$ is the global background RMS. Table 1 gives the number of the retrieved sources from the modelled catalogue in magnitude bins and the number of spurious detections. This number increases rapidly when the detection threshold is lowered, see Figure 1 for a summary of the different tests. On the other hand, by selecting a higher threshold, the number of extracted real sources decreases. A compromise value for the detection threshold on our narrow band image is $0.9 \sigma$, because the differential decrease of the number of retrieved real objects for detection threshold above $0.9 \sigma$ is always larger than the increase in the number of spurious detections, as it is evident from Table 1 and Figure 2. For the source extraction in the on and off-band images, we decided then to use a detection threshold of $1.0 \sigma$, to be conservative.

We can compute the limiting magnitude for the catalogue extracted from our narrow band image as the magnitude for which $50 \%$ of the input objects are retrieved; the limiting magnitude is 24.2 with a $1.0 \sigma$ threshold. The estimated line fluxes for ICPNe in Virgo cluster empty fields are in the range $25.8<m(5007)<27.5$ (Feldmeier et al. 1998). This magnitude interval corresponds to $23.3<m_{A B}<25.0$ and thus our limiting magnitude allows us to detect the first magnitude

\footnotetext{
${ }^{6}$ In this work the optimum parameter set for extracting catalogues was obtained through a complete image simulation, i.e. background plus modeled catalogue. The background was produced using the interpolated background image and noise from the background-rms map, both produced by SExtractor as check-images (see SExtractor manual for additional details). To this background simulated image, we added the modeled catalogue of point-like sources.
} 
interval of the ICPNe luminosity function.

Emission line objects will be extracted from a master catalogue produced as follows:

- detect and measure magnitudes in a fixed aperture ${ }^{7}$ for all objects in the [OIII] narrow band image,

- perform aperture photometry in the $\mathrm{V}$ band image at the $(\mathrm{x}, \mathrm{y})$ positions of the detected [OIII] sources, with SExtractor in dual image mode (see SExtractor manual for additional details).

The narrow band and V band image are registered before catalogue extraction. The ICPNe candidates are identified as those point-like objects with $m_{n}-m_{v}<<0$ and no detected continuum. The objects with no detected continuum are those whose measured $\mathrm{V}$ mag(aper) values lie below the $1 \sigma$ threshold above sky; for our RCN1 V band image this corresponds to 24.75 mag.

\section{Catalogues of emission line objects and spectroscopic validation}

We extracted a catalogue of objects in the [OIII] image field RCN1 as described in Section 3.1, for which we have aperture and Kron magnitudes in [OIII] $\left(m_{n}\right)$ and V band $\left(m_{v}\right)$. Figure 3 shows the color magnitude diagram (CMD) for the 18178 objects detected in our field. Most of them are continuum objects with an average $m_{n}-m_{v}$ color around 0 . There is some intrinsic scatter in the CMD, even with the two filters being so close in wavelength: the sequence of blue and red stars can be seen in the magnitude interval $m_{n}=16 \div 22$. The scatter in the colors of continuum objects at fainter magnitudes is caused by two factors: contaminations by line-emitters and background field galaxies plus errors in the photometry for faint objects. Moreover most faint galaxies are red, and scatter up in the diagram; we are interested in emission line objects whose color in $m_{n}-m_{v}$ is negative. ICPNe candidates are not the only emission line objects with this property. This color criteriom will in principle select at least the following four different kinds of emission line objects: ICPNe and HII regions in Virgo, $[\mathrm{OII}] \lambda=3726.9 \AA$ emitters at $\mathrm{z} \approx 0.34$, Ly $\alpha$ galaxies at $\mathrm{z} \approx 3.1$. The contamination from [OII] emitters can be greatly reduced by considering only objects with observed equivalent width (EW) greater than $100 \AA$. Colless et al. (1990), and recently Hammer et al. (1997) and Hogg et al. (1998) found no [OII] emitters at $\mathrm{z}=0.35$ with rest frame EW greater than $70 \AA$, which implies an observed EW of $95 \AA$. Following Teplitz et al. (2000), if the emission line is a negligible contribution to the broad band flux, as it is in this case, the observed equivalenth width is

$$
\mathrm{EW}_{o b s} \sim \Delta \lambda_{n b}\left(10^{0.4 \Delta m}-1\right)
$$

\footnotetext{
${ }^{7}$ For aperture photometry, we set a radius equal to $1.5^{\prime \prime}=3 \sigma$, where $\sigma=\frac{F W H M}{2.356}$. If a detected source is point-like, a $6 \sigma$ diameter aperture contains $98.89 \%$ of the total flux.
} 
where $\Delta \lambda_{n b}$ is the width of the narrow band filter in $\AA$. Objects with observed $\mathrm{EW}=95 \AA$ are those with color $m_{n}-m_{v}=-0.91$ : our emission line candidates will be selected according to the condition $m_{n}-m_{v}=-1.0$ corresponding to an observed $\mathrm{EW}=110 \AA$.

Due to the photometric errors at faint magnitudes, objects with intrinsic $m_{n}-m_{v}<-1.0$ do not all fall above the corresponding straight line in the CMD. The scatter at faint magnitudes can mimic emission line objects from faint continuum sources.

To determine the effects of these photometric errors quantitatively, we modelled a population of point-like sources with intrinsic colors $m_{n}-m_{v}=0$ and -1 , and studied their distribution in the CMD. The continuous lines over plotted in Figure 3 represent the $99 \%$ and $99.9 \%$ lines for a distribution of modelled objects with color $m_{n}-m_{v}=0$. Thus, above these lines will fall $99 \%$ and $99.9 \%$ of all continuum objects. The dashed lines in Figure 3 represent the $1 \times \mathrm{RMS}$ and $2 \times \mathrm{RMS}$ lines of the color distribution for objects with $m_{n}-m_{v}=-1$. If the scatter of these objects in the CMD plane is Gaussian, then $84 \%$ and $97.5 \%$ of these objects are located above these lines.

The "on-off" techniques for the identifications of ICPNe requires that there be no detected emission in the off-band image, which therefore needs to reach to fainter limiting AB magnitude to avoid contamination of our sample by faint continuum objects. The diagonal line in Figure 3 represents the $\mathrm{V}$ magnitude for fluxes corresponding to $1.0 \times \sigma$ above sky in the off-band. Objects above this diagonal line display some emission in $\mathrm{V}$. Objects whose $\mathrm{m}_{v}$ fall below this line are not detected by SExtractor ${ }^{8}$ on the off-band RCN1 image, with the selected low-threshold for detection. Thus ICPNe candidates must be objects located in this region. SExtractor in dual image mode cannot compute an $m_{v}$ magnitude (either mag(aper) or mag(auto)) for some of the [OIII] detected sources: these sources will all be considered as emission line candidates.

\subsection{Point-like and extended objects}

The typical size of a PN is about $0.1-1 \mathrm{pc}$; therefore at the distance of the Virgo cluster $(\sim 15$ $\mathrm{Mpc}$ ) they cannot be resolved on our images. Two methods were used to test whether an object is extended or point-like: (1) a 2D Gaussian fit and comparison to the PSF and (2) comparison between mag(auto) and mag(aper) magnitudes from SExtractor (see Section 3.1).

\subsubsection{Point-like objects: 2D Gaussian fit}

The stellar PSF for our images can be modelled with a two dimensional Gaussian. Twenty un-saturated stars were selected in the narrow band image and a 2D Gaussian fit was produced

\footnotetext{
${ }^{8}$ Their magnitudes are computed from aperture photometry in a $3^{\prime \prime}$ diameter circular aperture at the positions of the $[\mathrm{OIII}]$ sources.
} 
with the task FITPSF within IRAF. Our emission line candidates are faint, and their fluxes in the PSF wings looks as background noise; therefore we modeled the light distribution in our selected emission line candidates using a 2D Gaussian. Those objects with $\sigma_{\text {object }}-\sigma_{P S F}>>0$ are most probably extended objects, while those with $\sigma_{\text {object }}-\sigma_{P S F} \sim 0$ are most likely point like objects. Because our candidates are faint, we need to test whether noise and small changes of the PSF across the wide field can affect their light distribution in a systematic way. We simulated sources with $\sigma_{\text {object }}=\sigma_{P S F}$ and [OIII] magnitude in the range from 20 to 25 . These simulated objects were added onto the narrow band image and a 2D Gaussian was fitted in the same way as for the real objects. The results are shown in Figure 4; the dashed lines over plotted in the upper panel of Figure 4 show the maximum and minimum value of $\sigma_{\text {object }}-\sigma_{P S F}$ in each $\mathrm{m}_{n}$ magnitude bin of 200 modelled objects. The range in $\sigma_{\text {object }}-\sigma_{P S F}$ is larger for fainter objects, where the noise affects their 2D light distribution. Based on this simulation, point-like objects are those candidates whose $\sigma_{\text {object }}-\sigma_{P S F}$ lies between the dashed lines in the upper panel of Figure 4 . Those objects

whose $\sigma_{\text {object }}-\sigma_{P S F}$ happen to fall below the dashed-line curve, turned out to be bad pixels or have cosmic-rays residual in the area influenced by the CCD gaps, where fewer frames were averaged, and were not plotted. They were rejected in the final catalogue.

\subsubsection{Point-like objects: magnitude selections}

An independent test on whether a source is extended or not is based on the mag(auto)mag(aper) vs. $\operatorname{mag}($ auto) diagrams for the detected sources. In our extraction procedure the aperture magnitude mag(aper) is computed in a fixed aperture of diameter $1.5^{\prime \prime}$, equivalent to $3 \times \sigma$ of the mean seeing measured on the image. The so-called mag(auto) is the same to 0.1 mag as mag(aper) for point-like objects, while the two magnitudes differ for extended objects.

For the point-like objects modelled in our Monte-Carlo simulations, we studied the distribution of mag(auto)-mag(aper) as a function of the object magnitude mag(auto). This is shown in the lower panel of Figure 4. As in the case of the 2D Gaussian fit, for bright point-like objects this difference is fixed and at faint magnitudes we have a larger spread because of photometric errors. Point-like objects are those which lie between the dashed lines of Figure 4.

The final set of point-like objects in our extracted catalogue is selected as those which satisfy both criteria: a 2D Gaussian fit which is consistent with the PSF, and mag(auto)-mag(aper) inside the distribution for simulated point sources. This is a necessary condition for our selected objects to be selected as point-like, but it is not sufficient, i.e. high redshift starbursts with a strongly peaked central luminosity may still get into our sample. 


\subsection{Spectroscopic confirmation}

For spectroscopic validation, we use a sample of spectra obtained with the $2 \mathrm{dF}$ fiber spectrometer at the $4 \mathrm{~m}$ Anglo Australian Telescope by Freeman et al. (2000; 2001, in prep.). Spectra were acquired with a spectral resolution $\mathrm{R}=2000$ in the wavelength range $4550 \AA$ to $5650 \AA$ and a total integration time of $5 \mathrm{hrs}$ on the 15th of March 1999.

A total of 43 fibers were allocated to the narrow-line emitter candidates selected by Feldmeier et al. 1998 at coordinate $\alpha(J 2000)=12: 30: 39$ and $\delta(J 2000)=12: 38: 10$; we will refer to this field as the FCJ field. In this field, 15 objects were spectroscopically confirmed as emission line objects. The best S/N spectra of the ICPNe candidates in the FCJ field are shown in Figure 5. As a further test on the intrinsic nature of these objects, 23 spectra of ICPNe candidate from the whole area in Virgo surveyed with the 2dF (see Freeman et al. 2000 for additional details), were summed: these 23 objects include the 15 from the FCJ field and another 8 from other Virgo intracluster empty fields. The cumulative spectrum shows the [OIII] doublet very clearly, with the expected equivalent width ratio $E W_{5007}=3 \times E W_{4959}$ for the [OIII] doublet (see Figure 5). Of the 15 spectroscopically confirmed objects in the FCJ field, 13 are ICPNe and 2 are broadlined Ly $\alpha$ emitters. The broad-lined sources were identified as Ly $\alpha$ by Freeman et al. (2000) and Kudritzki et al. (2000) because of their typical asymmetric line-profile; furthermore in Kudritzki et al. (2000) no $\mathrm{H} \beta$ and [OIII] emissions were detected at the expected redshifted wavelengths, under the hyphothesis that the strongest emission was [OII] at redshift 0.35 .

These observations show that a significant fraction of emission line candidates selected carefully with the "on-off" band technique are indeed ICPNe. They were used to make the first kinematical study of the ICPNe population in Virgo. These issues will be discussed by Freeman et al. (2001, in preparation). Here these objects are used for validation of the automatic selection procedure.

Validation of the selection procedure To compare our photometric selection of emission line objects with the spectroscopic confirmations, we have applied our selection procedure to the "onoff" images for the FCJ field. The survey on the FCJ field was carried out with a $44 \AA$ narrow band [OIII] filter, with central wavelength at $\lambda_{c}=5027 \AA^{9}$. Of the 43 emission line candidates in FCJ selected with the blinking technique and allocated a fiber in the spectroscopic follow-up, we could detect and measure narrow band magnitudes in the images for 36 of them. The remaining 7 objects were very near to bright objects and were therefore discarded because of likely errors in the SExtractor photometry. Of these 7 objects, 4 were spectroscopically confirmed (3 ICPNe + one Ly $\alpha$ ). In the following, we discuss the percentage of spectroscopically confirmed objects in the sample of 36 objects with allocated fiber which are in common with our selected sample. In this sample, 11 objects (i.e. 30\%) are spectroscopically confirmed as emission line objects; of these objects 10 are confirmed ICPNe and one is a Ly $\alpha$ candidate.

\footnotetext{
${ }^{9}$ The conversion from $m_{A B}$ to $m_{5007}$ for this filter is $m_{5007}=m_{A B}+3.04$.
} 
In Figure 6 we show the CMD for all sources in this field. Squares indicate those sources in common with Feldmeier's catalogue of emission line objects with an allocated fiber, while asterisks indicate those ICPNe candidates with confirmed spectra; the Ly $\alpha$ is indicated with a full square. The lines plotted in Figure 6 have the same meaning as in Figure 3. For 16 out of the 36 allocated fiber, SExtractor could not detect a $m_{v}$ magnitude at the $x, y$ position of the [OIII] detected emission. All these objects would be selected and are reported on this diagram assuming a $m_{v}=27^{10}$. Two out of the 10 confirmed ICPNe were in the region of the frame with bright scattered light from nearby luminous objects. So their $\mathrm{V}$ magnitude appear above the detection limit, because of scattered background light. The 8 out of the 10 confirmed ICPNe and the Ly $\alpha$ emitter are found

- below the line for detection in the off-filter,

- below the $97.5 \%$ line of objects with colour $=-1^{11}$.

- or in the sample with no detected off-band mag(aper), when SExtractor is used in dual image mode.

The sample selected according to these criteria has a higher spectroscopic confirmation rate, $50 \%$, compared to the Feldmeier's sample for the FCJ field, i.e. 30\%. The part of the 36 objects which do not satisfy the above criteria have a much lower spectroscopic confirmation rate: $2 / 18=10 \%$. These percentages are lower limits because, as Fig 5 shows, they are based on low signal-to-noise spectra.

We also ran the morphological selection procedure on the spectroscopically confirmed emission line objects, which are in common with our photometrically selected sample. Eight objects which showed an unresolved [OIII] $\lambda 5007 \AA$ emission and the additional $\lambda 4959 \AA$ line are point-like sources. These are the confirmed ICPNe. One object which is spectroscopically confirmed as an emission line object with a broad asymmetric emission line (see Figure 5) is classified as extended, and it is probably a Ly $\alpha$ emitter.

Based on the spectroscopically confirmed sample, our selection criteria for Bona Fide ICPNe candidates are the following: these must be point-like objects lying below the $97.5 \%$ line for intrinsic color $m_{n}-m_{v}<-1$, and with no detection in the off-band (V) image. This is the population of objects that we discuss hereafter in the RCN1 field. From Figure 4, we found 75 point-like objects with those characteristics. These are the ICPNe candidate in our RCN1 field.

\footnotetext{
${ }^{10}$ Here we adopted the $\mathrm{V}$ magnitude that a star would have if the flux from a [OIII] emission of $m_{A B}=24.5$ would have been seen through a $\mathrm{V}$ band filter

${ }^{11}$ For the FCJ filter, this corresponds to an $\mathrm{EW} \sim 70 \AA$.
} 


\section{The Virgo cluster ICPNe}

The observations presented in this work allow us to obtain a large statistical sample of IPNe candidates in the RCN1 field. Using also the data from the FCJ field, we can make a preliminary study of their distribution on the sky.

\subsection{Luminosity function and cluster depth}

The PNLF has been used extensively as a distance indicator. Observations in ellipticals, spirals and irregular galaxies have shown a PNLF truncated at the bright end. The shape of this LF is given by the semi-empirical fit:

$$
N(M)=c_{1} e^{c_{2} M}\left[1-e^{3\left(M^{*}-M\right)}\right]
$$

where $c_{1}$ is a positive constant, $c_{2}=0.307$ and the cut-off $M^{*}(5007)=-4.5$ (Ciardullo et al. 1989). The evidence for a bright cut-off comes from the observations of nearby galaxies, and its dependence on the age and metallicity of the stellar population was analysed in some detail by (Dopita et al. 1992; Méndez et al. 1993). Its overall metallicity dependence seems to be modest provided that the metallicity differences are no more than about 30\%, but see also Méndez et al. (1993) for an elaborate discussion of the effects of sample size and population age.

Figure 7 shows the luminosity function (LF) of the spectroscopically confirmed ICPNe from the FCJ field, with the best fit $\mathrm{c} 1$ and distance modulus values computed for a single population of $\mathrm{PNe}$ at a fixed distance ${ }^{12}$, using a $\chi^{2}$ minimization test. The fit was done in the first magnitude interval (first three bins) where the sample is complete. The best-fit distance modulus is 30.53, once the empirical PNLF is convolved with the photometric errors. On the same plot, we show the PNLF which is expected for a distance modulus of 30.86, as determined from PNe in the inner part of M87 (Ciardullo et al. 1998). Because metallicity and age effects do not strongly affect the bright cut-off (Dopita et al. 1992), the most probable interpretation of Figure 7 is that the spectroscopically confirmed ICPNe in the FCJ field are closer to us than the M87 PNe. When a large area is surveyed in Virgo empty fields, we preferentially pick up those ICPNe which are nearer to us, i.e. brighter. Thus the inferred front edge of the ICPN population in the FCJ field is at $14 \%$ shorter distance than M87, which corresponds to $2.1 \mathrm{Mpc}$, when the distance to M87 is at 14.9 Mpc. Ciardullo et al. (1998) and Feldmeier et al. (1998) already suggested that the LF of ICPNe may be distorted because of the Virgo cluster depth. However, we have now spectra which confirm this hypothesis.

Figure 8 shows the PNLF for the ICPNe candidates from the RCN1 field. Here again the cut-off is at brighter magnitudes than for the PNe in M87. Could this be due to contamination

\footnotetext{
${ }^{12}$ The $m_{5007}$ magnitudes are from Feldmeier's 1998 data.
} 
by Ly $\alpha$ emitting objects at $z \sim 3.13$, which are also picked up by our technique? There is no well-sampled Ly $\alpha$ LF of such objects available in the literature. We have therefore estimated the LF of Ly $\alpha$ emitters in our sample from the work of Steidel et al. (2000). These authors showed that the continuum LFs of Ly $\alpha$ emission selected galaxies and of field Lyman break galaxies (LBGs) are consistent with being the same, and that there is no evidence for enhanced Ly $\alpha$ emission from fainter galaxies. The equivalent width (EW) distribution of their spectroscopic sample is consistent with being a Gaussian centered at zero, such that the fraction of objects with EW $>80 \AA$ (restframe EW $>20 \AA$ ) is $\simeq 25 \%$. We can thus estimate the narrow band magnitude LF of Ly $\alpha$ emitters in our on-band filter as follows. We take the LF of field LBGs from Steidel et al. (1999) with the parameters given in their Fig. 8 except $\mathrm{m}_{*}^{\mathrm{V}}=\mathrm{m}_{*}^{\mathrm{R}}+0.24$ (as LBGs have about $20 \%$ fainter flux density in V than in R; M. Giavalisco, private communication). We then apply the same selection criteria to those sources as to our ICPNe candidates, that $\mathrm{m}_{\mathrm{V}}=24.75$, and finally convolve with the EW distribution for the Ly $\alpha$ emission, accepting only those galaxies with an observed EW> $110 \AA$ which correspond to our sample selection criteria. The resulting LF, scaled to our larger field and effective volume, is shown as the full line in Fig. 8.

We have also added the observed data points constructed from the Ly $\alpha$ blank field search done by Cowie \& Hu (1998), as this survey is carried out in a very similar way to our survey for ICPNe in the Virgo cluster fields. We used their V magnitudes and EWs listed in their Table 1 for those objects whose $\mathrm{V}$ magnitudes are fainter then 24.75, and then converted to our filter AB magnitudes. Once the resulting LF is scaled to our larger field, the Cowie \& Hu (1998) narrow band LF for the Ly $\alpha$ emitters agrees very well with that computed from Steidel et al. (2000). The comparison of the candidate ICPNe LF in the RCN1 field with the computed narrow band LF of the field Ly $\alpha$ emitters from Steidel et al. (2000) and Cowie \& Hu (1998) show that the Ly $\alpha$ contribution does not dominate in the narrow band $\mathrm{AB}$ magnitude range 23-24: the fraction of expected contamination by Ly $\alpha$ emitters with $\mathrm{V}$ magnitude fainter of 24.75 in the first magnitude bin is $15 \%$. This is in agreement (within the errors) with the fraction of Ly $\alpha$ contaminants of $25 \%$, as obtained from the spectroscopic sample

Therefore most over-luminous bright objects in the RCN1 are likely to be ICPNe, and they are brighter than those at the cut-off in the M87 PNLF because they too are closer to us than M87. Once we have convolved the empirical PNLF given by Equation 5 with the photometric error of our sample, we fit the data point of RCN1 LF and obtain the best fit distance modulus of 30.29 with the following errors ${ }_{-0.2}^{+0.2}$, which refers to the $90 \%$ c.l.. Our estimate of the distance modulus is slightly biased from the contribution of the [OIII] emission at $4958.9 \AA$ which enters in our filter band pass for radial velocities $v_{\text {rad }}>1580 \mathrm{~km} \mathrm{~s}^{-1}$. We have estimated this systematic contribution assuming a Gaussian velocity distribution for the ICPNe in Virgo (with $v_{\text {mean }}=1100 \mathrm{~km} \mathrm{~s}^{-1}$ and $\sigma=800 \mathrm{~km} \mathrm{~s}^{-1}$ ). This gives a maximal brightening of the PNLF of 0.13 mag, assuming that most ICPNe at the front edge of the cluster have the same velocity dispersion as galaxies in Virgo. If this systematic brightening is taken into account, the distance modulus to the front edge of the Virgo cluster is $30.4 \pm 0.2$ at RCN1, in agreement with the distance modulus derived for the spectroscopic 
sample, within the photometric errors. This implies that the front edge population seen in the RCN1 Virgo field is at $19 \%$ shorter distance than M87, i.e. $2.8 \mathrm{Mpc}$ in front of the cluster center. The Virgo cluster appears to have a significant depth.

Previously, Yasuda et al. (1997) have argued that the distribution of spiral galaxies in Virgo is best described as an elongated, cigar-like structure with a depth $\pm 4 \mathrm{Mpc}$ from M87. Neilsen \& Tsvetanov (2000) and West \& Blakeslee (2000) have used surface brightness fluctuations to determine the distances to the Virgo ellipticals, and found that most of these are arranged in a nearly collinear structure extending about $\pm(2-3)$ Mpc from M87. Therefore our result from the ICPNe population is not entirely surprising.

\subsection{Luminosity surface density}

From our results we can determine a surface brightness associated with the diffuse population of evolved stars in the FCJ and the RCN1 fields. Given our tight selection criteria in the photometric analysis and the likely incompleteness of the $2 \mathrm{dF}$ detections, this will in both fields be a lower limit. From stellar evolution theory, it is expected that the luminosity-specific stellar death rate of a galaxy should be independent of the precise state of the underlying stellar population. (Renzini \& Buzzoni 1986) have shown that this quantity is remarkably independent of both age and initial mass function: if this is the case, the number density of planetaries per unit bolometric luminosity should be approximately the same for every galaxy. Here we adopt the luminosityspecific planetary nebulae density from M31, $\alpha_{1, B}=9.4 \times 10^{-9} \mathrm{PNe} L_{B \odot}^{-1}$ (Ciardullo et al. 1989) to infer the amount of light associated with the ICPNe. ${ }^{13}$ For the spectroscopically confirmed sample in the FCJ field, the inferred luminosity surface density is $5.2 \times 10^{6} L_{B \odot} \operatorname{arcmin}^{-2}$, corre-

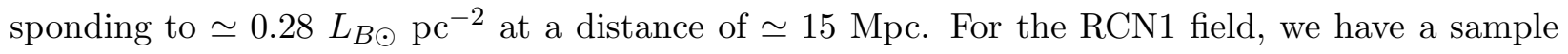
of 75 candidates. From the spectroscopic follow-up by Freeman et al. (2000) the contamination by high redshift objects can still be up to $25 \%$, and the possibility of large-scale structures in the Ly $\alpha$ emitters implies some uncertainties in a simple statistical subtraction for ICPN measurements. Removing $25 \%$ of our sources gives a likely lower limit to the surface luminosity density of the ICPNe population. Thus we take the number of ICPNe in this field to be 56 , which on an area of $31.8 \times 30.1$ $\operatorname{arcmin}^{2}$ amounts to a luminosity surface density of $6.2 \times 10^{6} L_{B \odot} \operatorname{arcmin}^{-2}$, which corresponds to $\simeq 0.33 L_{B \odot \mathrm{pc}^{-2}}$, similar to that for the spectroscopically selected sample. The inferred blue surface brightness is $\mu_{B}=28 \mathrm{~m}_{B} \operatorname{arcsec}^{-2}$. The inferred luminosity derived assuming all the sample at a single distance gives the smallest amount of intracluster light: from Feldmeier et al. (1998), if the ICPN were spherically distributed with a constant density, the amount of starlight could be up to a factor of three larger.

\footnotetext{
${ }^{13}$ The most appropriate value of $\alpha_{1, B}$ for the intracluster light is unknown at this stage, therefore we adopted the $\alpha_{1, B}$ for the bulge of M31, which is an evolved population similar to those in early-type galaxies. Independent measurements of $\alpha_{1, B}$ in ellipticals shows a variation up to a factor 5 .
} 
The result that the inferred intracluster surface brightnesses for both fields are similar is robust and very interesting, because the FCJ field is $45^{\prime}$ from the center of the cluster core, while the RCN1 field is $1.5 \mathrm{deg}$ from the center. The azimuthally averaged total luminosity density contributed by galaxies is given by Binggeli et al. (1987) as a function of distance to the core center. This function has a large scatter (see Figure 16 (Binggeli et al. 1987)), and implies $\sim 10^{11} \mathrm{~L}_{B \odot} \mathrm{deg}^{-2}$ at the FCJ distance $\left(45^{\prime}\right)$ and $\sim 3 \cdot 10^{10} \mathrm{~L}_{B \odot} \mathrm{deg}^{-2}$ at the RCN1 distance $(1.5 \mathrm{deg})$.

Over the range of radii probed by these two fields, the luminosity surface density of galaxies in Virgo decreases by a factor of $\sim 3$ (Binggeli et al. 1987) while that for the ICPNe population is nearly constant. The diffuse light contributes from $17 \%$ (FCJ) to $43 \%$ (RCN1) of the total (i.e. galaxies + diffuse light) luminosity density in these two fields (recall that these numbers are lower limits from our data). Because we have no evidence for a radial gradient in the ICPNe luminosity profile, it is very difficult to estimate the total luminosity for this population at this stage.

\section{Conclusions}

We have imaged an empty area of $34 \times 34 \operatorname{arcmin}^{2}$ in the Virgo cluster in a narrow band filter centred at the redshifted Virgo [OIII] emission and in the $\mathrm{V}$ band. The area surveyed in this work is the largest probed for ICPNe in one single pointing so far, and it allows us to get a better statistical sample of ICPNe. We have developed an automatic extraction procedure for ICPNe candidates using publicly available photometry software (SExtractor). This selection procedure was validated using a spectroscopic confirmed sample of ICPNe. In our field 75 objects were found which satisfy the selection criteria for ICPNe, i.e., point-like, no measurable continuum, and equivalent width EW > $110 \AA$ after error convolution.

The luminosity functions for both the spectroscopically selected sample and the ICPNe sample in the new field show a brighter cut-off compared to the M87 PNe. We have evaluated the possible contribution of Ly $\alpha$ emitting objects at redshift $z=3.13$ from Steidel et al. (2000) and found that these objects contribute mostly at 1-2 magnitudes fainter than the bright cutoff found here, and in any case only up to $25 \%$ of the total sample. The bright cutoff is therefore most probably due to the three-dimensional depth of the Virgo cluster: our results place it at $14 \%$ - $19 \%$ shorter distance than M87. Our selected sample traces preferentially the edge of the cluster near us.

The luminosity surface density in these two fields is about $6 \times 10^{6} L_{B \odot} \operatorname{arcmin}^{-2}$ with both fields having similar densities. The lack of radial gradient between both fields implies that the total amount of luminosity in the diffuse component is very sensitive to the outer cut-off, and could be comparable to the luminosity in galaxies.

The authors wish to thank the referee, Dr. R. Ciardullo, for his insightful comments. Moreover we would like to thank the ESO 2.2m telescope team for their help and support during the observations carried out for this project, in particular E. Pompei and H. Jones. We thank F. Valdes for his 
help and support in using MSCRED in reducing WFI mosaic data, the OACDF team for support during astrometry and co-addition, and E. Bertin for his help and suggestions in using SExtractor. The authors acknowledge the use of NED and the CDS public catalogues. JALA and OG were supported by the grant 20-56888.99 from the Schweizerischer Nationalfonds. NRN was supported by the European Social Funds and acknowledges the support from the University Federico II for travel grants within the program for International Exchange.

\section{A. $m(5007)$ and the AB magnitude system}

Absolute flux calibrations for our narrow band imaging (with an interference filter centred on the [OIII] redshifted wavelength at the Virgo systemic velocity) were performed according to Jacoby et al. (1987). These calibrations give us the total flux in the [OIII] line in ergs $\mathrm{sec}^{-1} \mathrm{~cm}^{-2}$, which we indicate as $F_{5007}$. Following Jacoby (1989), the m(5007) magnitudes are defined as:

$$
m(5007)=-2.5 \log F_{5007}-13.74
$$

The magnitude in the $\mathrm{AB}$ system are given by:

$$
m_{A B}=-2.5\left(\log F_{\nu}+19.436\right)
$$

and we can rewrite $\mathrm{m}_{A B}$ as function of $\mathrm{m}(5007)$ as follows:

$$
m_{A B}=-2.5 \log F_{5007}-2.5 \log \left(\frac{\lambda_{c}^{2}}{\Delta \lambda_{e f f} c}\right)-48.59=m(5007)-2.51
$$

where

$$
\Delta \lambda_{e f f}=\int d \lambda T(\lambda)
$$

$\lambda_{c}$ is the filter central wavelength, and $T(\lambda)$ is the filter bandpass. 


\section{REFERENCES}

Arnaboldi, M., et al. 1996, ApJ, 472, 145

Bernstein, G.M. et al. 1995, AJ, 110, 1507

Bertin, E. \& Arnouts, S. 1996, A\&AS 117, 3993

Binggeli, B., Tammann, G.A., Sandage, A. 1987, AJ, 94, 251

Calcáneo-Roldán, C., Moore, B., Bland-Hawthorn, J., Malin, D., Sadler, E.M. 2000, MNRAS, 314, 324

Capaccioli, M., et al. 2001, A\&A, submitted

Ciardullo, R., Jacoby, G.H., Ford, H.C., Neill, J.D. 1989, ApJ, 339, 53

Ciardullo, R., Jacoby, G.H., Feldmeier, J.J., Barlett, R. 1998, ApJ, 492, 62

Colless, M., Ellis, R.S., Taylor, K., Hook, R.N. 1990, MNRAS 244, 408

Cowie, L.L., \& Hu, E.M. 1998, AJ, 115, 1319

Crane, P., Tammann, G.A., Woltjer, L. 1977, Nature, 265, 124

Dopita, M.A., Jacoby, G.H., Vassiliadis, E. 1992, ApJ, 389, 27

Durrell, P., Ciardullo, R., Feldmeier, J., Jacoby, G.H. 2001, ApJ, submitted

Feldmeier, J.J., Ciardullo, R., Jacoby, G.H. 1998, ApJ, 503, 109

Feldmeier, J.J. 2000, Ph D. Thesis, Penn State University

Ferguson, H., Tanvir, N.R., von Hippel, T. 1998, Nature, 391, 461

Freeman, K.C., et al. 2000, ASP Conf. Series 197, 389

Hammer, F., et al. 1997, ApJ, 481, 49

Hogg, D.W., Cohen, J. G., Blandford, R., Pahre, M. A. 1998, ApJ, 504, 622

Hui, X., Ford, H.C., Ciardullo, R., Jacoby, G.H. 1993, ApJ414, 463

Guldheus, D.H. 1989, ApJ, 340, 661

Kron, R.G. 1980, ApJ343, 305

Kudritzki, R.P., et al. 2000, ApJ536, 19

Jacoby, G.H., Quigley, R.J., \& Africano, J.L. 1987, PASP, 99, 672 

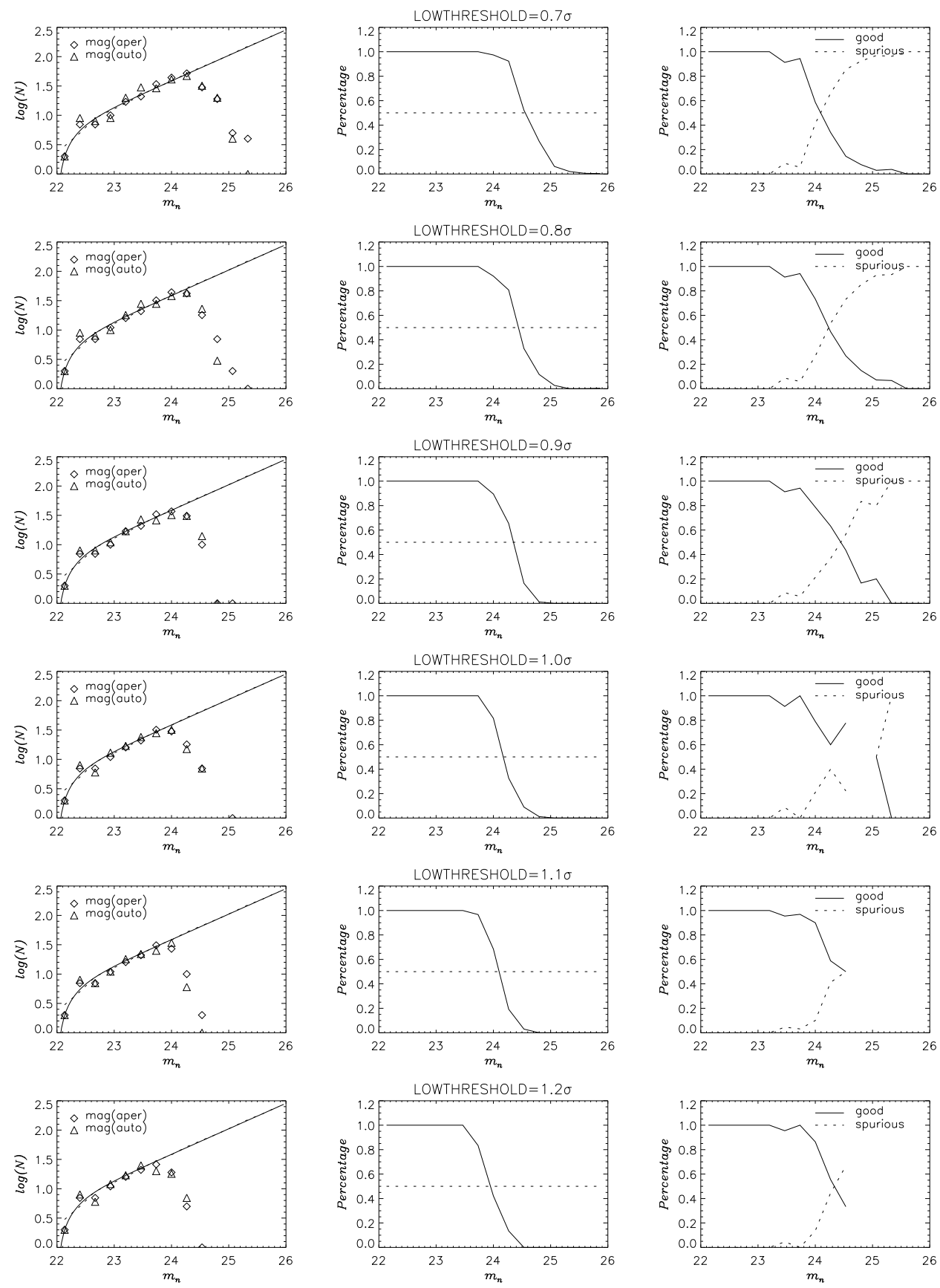

Fig. 1.- Monte Carlo simulations carried out for a modelled population of point-like objects. Left panels: the luminosity function adopted for the modelled objects (continuous line) and the recovered luminosity function (triangles-mag(auto) and diamonds-mag(aper)). Central panels: Percentage of modelled point-like objects recovered for the different detection thresholds used. The dotted line at $50 \%$ marks the completeness limit. Right panels: Percentage of the modelled point-like objects (continuous line) and of spurious objects (dashed line) normalised to the total extracted object catalogue in 0.25 magnitude bins. 


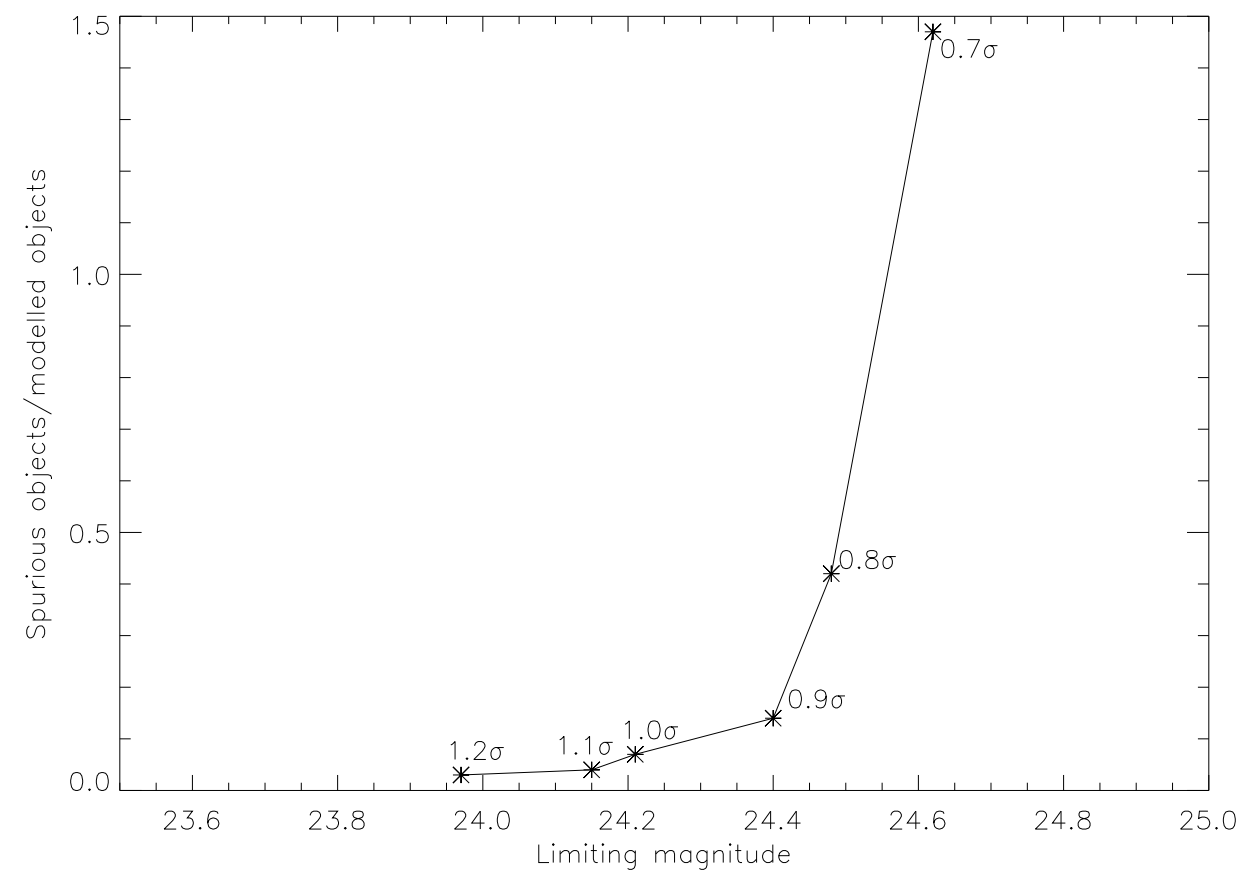

Fig. 2.- Ratios of spurious to real detections plotted as a function of limiting magnitude for different low thresholds. 


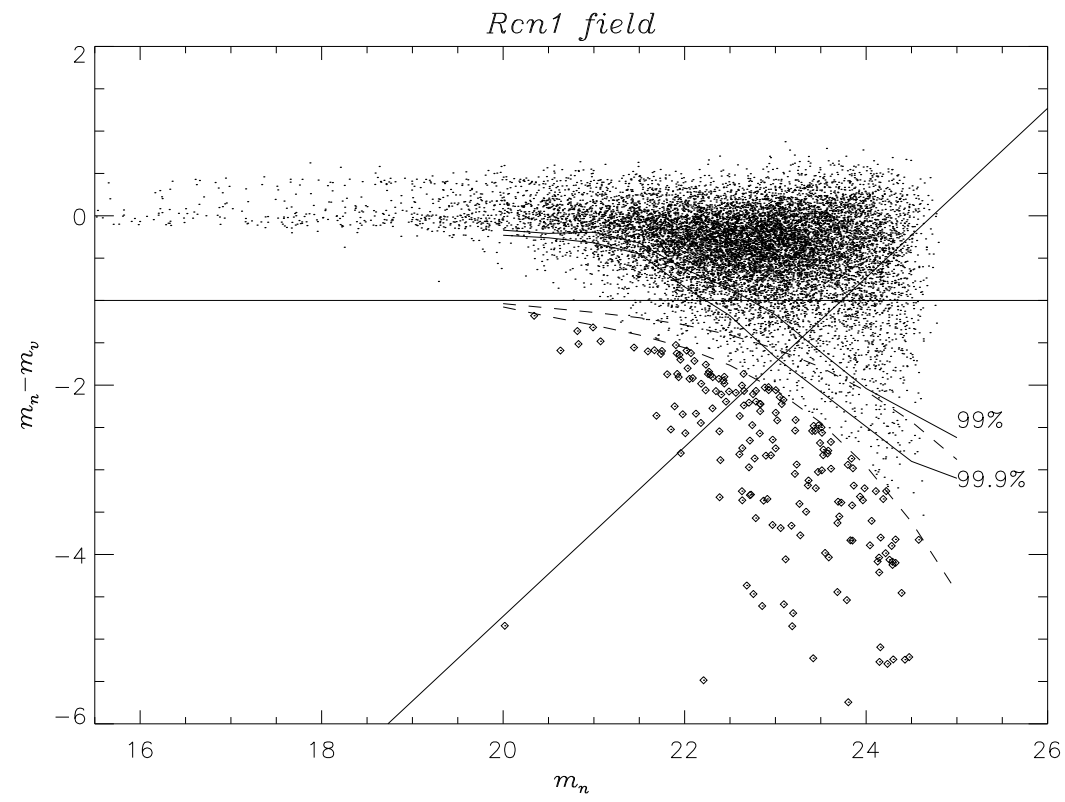

Fig. 3.- Color-magnitude diagram for all the sources in the RCN1 field. The horizontal line at $m_{n}-m_{v}=-1$ indicates objects with an observed $\mathrm{EW}=110 \AA$. The diagonal line shows the magnitude corresponding to $1.0 \times \sigma$ above sky in the V band. Full curved lines represent the $99 \%$ and $99.9 \%$ lines for the distribution of modelled continuum objects. The dashed lines represent $84 \%$ and $97.5 \%$ lines for the distribution of modelled objects with $m_{n}-m_{v}=-1$. The points are all objects detected by SExtractor. Diamonds are objects with a significant color excess in the narrow band filter. See text for details. 

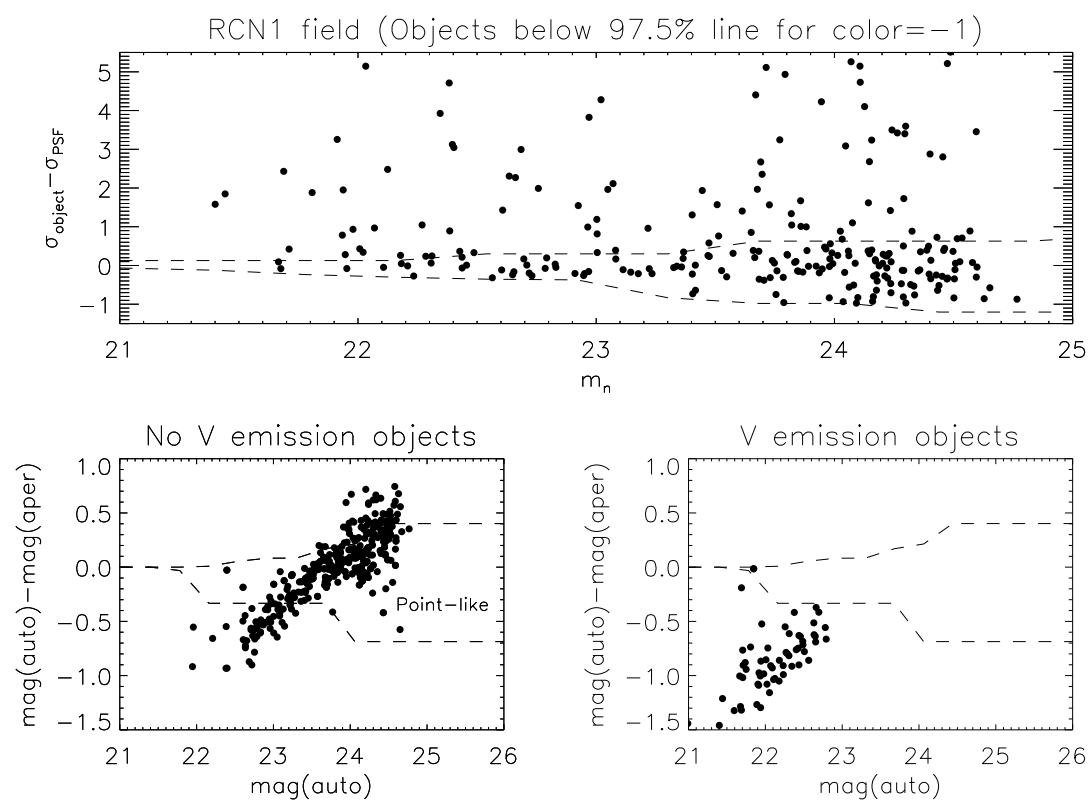

Fig. 4. - Top panel: $\sigma_{\text {object }}-\sigma_{P S F}$ vs. $m_{n}$ magnitude for catalogued emission objects. The dashed lines shows the $\sigma_{\text {object }}-\sigma_{P S F}$ boundaries for a simulated distribution of point-like objects. Bottom panel: mag(auto) - mag(aper) vs. mag(auto) of the objects selected in Figure 3. The dashed lines show the boundaries within which the distribution of modelled point-like objects falls. In both panels point-like emission objects are those which lie between the dashed lines. Objects with no emission in $\mathrm{V}$ are both point-like and extended (lower left panel), whereas objects with luminosity in $\mathrm{V}$ are all extended (lower right panel). 

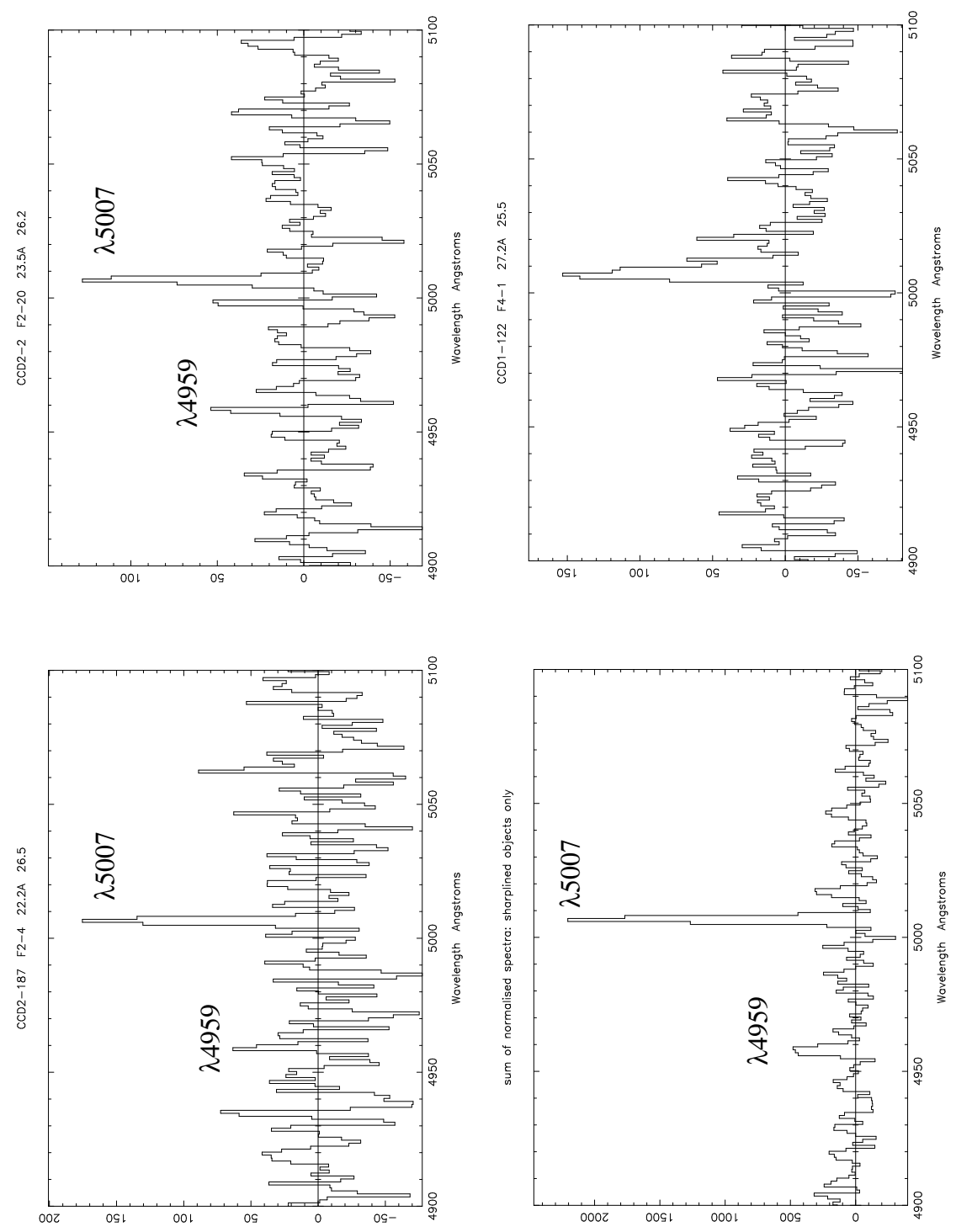

Fig. 5.- Top row: spectra of two ICPNe candidate from the AAT $2 \mathrm{dF}$ run. The $5007 \AA$ line is visible with $\mathrm{S} / \mathrm{N}>3$ and a second line at $4959 \AA$ corresponds to the second emission line of the [OIII] doublet. Lower left corner: cumulative spectrum of all $23 \mathrm{ICPNe}$ from the $2 \mathrm{dF}$ run. Each spectrum was shifted to zero velocity and normalized by the flux in the $5007 \AA$ [OIII] line. The equivalenth width ratio of the [OIII] $5007 \AA / 4959 \AA$ is $3.2 \pm 0.1$, so the real fraction of ICPNe in this sample is $0.94 \pm 0.03$ (Freeman et al. 2000, 2001 in preparation). Lower right corner: the $2 \mathrm{dF}$ spectrum of a likely Ly $\alpha$ emitter. The asymmetric profile of the line is clearly visible. 

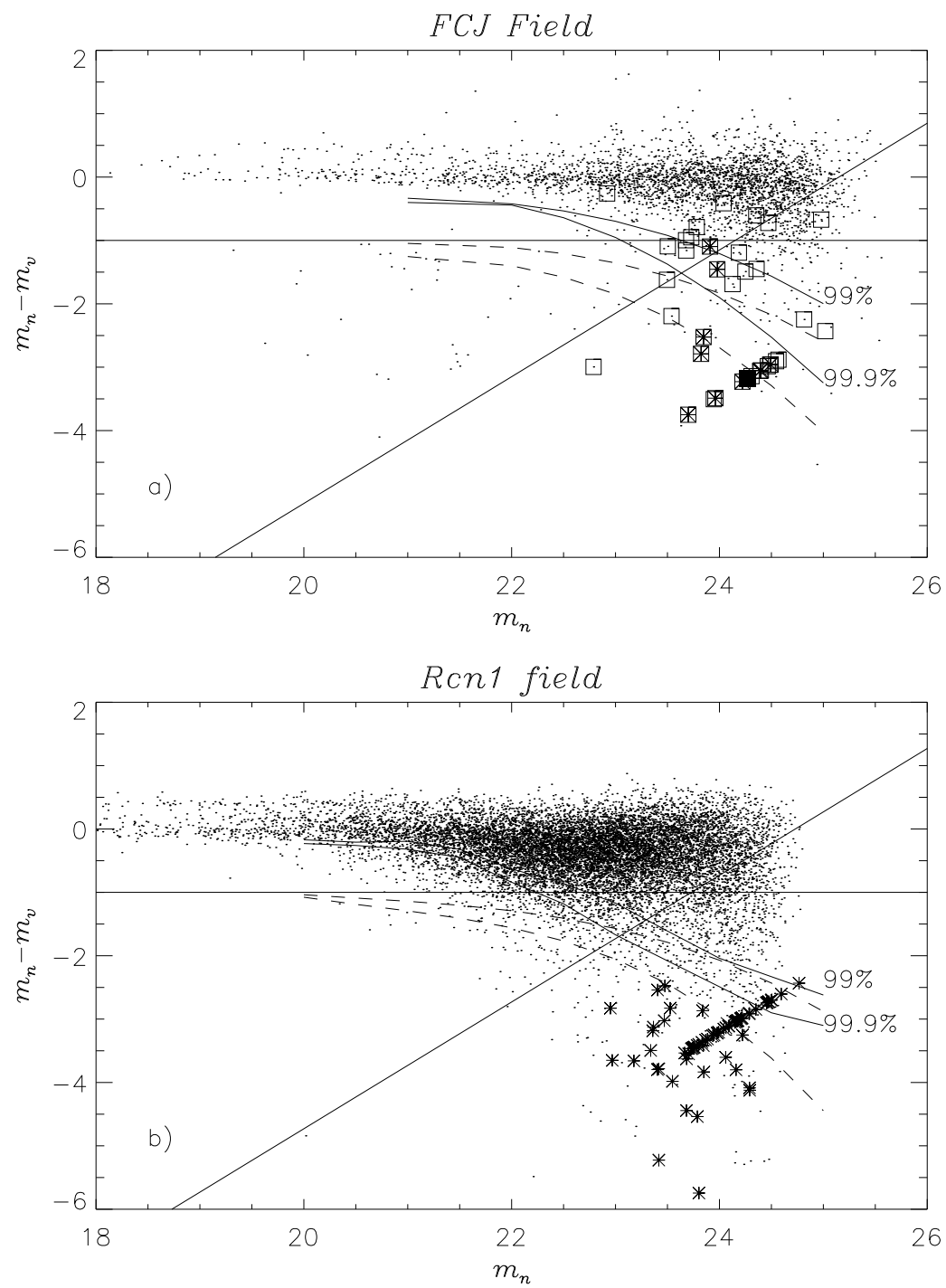

Fig. 6.- Color magnitude diagrams for the FCJ and the RCN1 fields. Upper panel: CMD for the FCJ field. The lines have the same meaning as in Figure 3. The diagonal line correspond to $m_{v}=24.75$. which is the $1.0 \times \sigma$ limiting magnitude in the $\mathrm{V}$ band. The over-plotted squares represent the allocated fibers of Freeman et al. (2000, 2001, in prep.) and the asterisks are the spectroscopically confirmed ICPNe. The full square represents a spectroscopically confirmed Ly $\alpha$ emitter located in this field. See text for details. Two spectroscopically confirmed PNe have off band fluxes near the limiting magnitude because of scattered light from bright emitters in the field. Fourteen objects in the Feldmeier et al. (1998) catalogue have no computed mag(aper) in the off-band, assigned by SExtractor in dual image mode: they have been assigned $m_{v}=27.0$ (see text). Lower panel: CMD for the RCN1 field. The ICPNe candidates are indicated with asterisks; those objects with no computed mag(aper) in the off-band, using SExtractor in dual image mode, have been assigned $m_{v}=27.0$. 


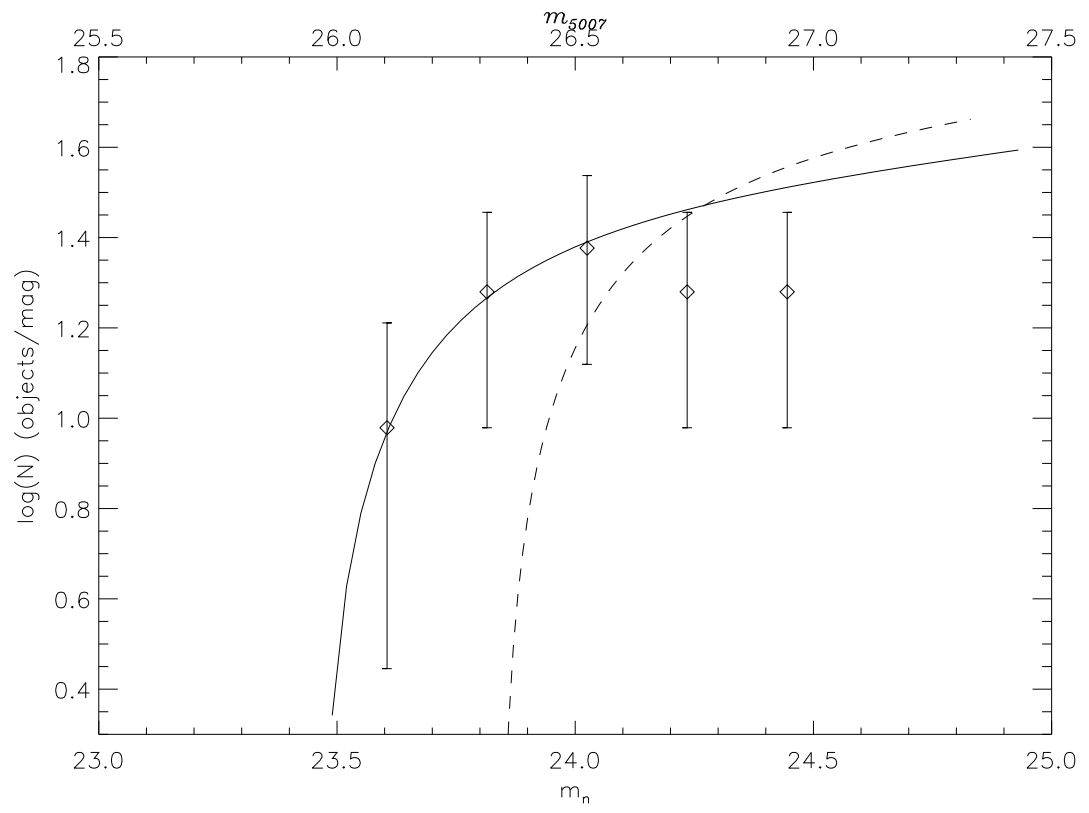

Fig. 7.- Luminosity function of the spectroscopically confirmed ICPNe from FCJ field. The continuous line is the best fit for a distance modulus equal to 30.53. The dashed lines indicates the same PNLF for the distance modulus 30.86 assigned to M87 using the PNLF within 20 arcmin of M87. 


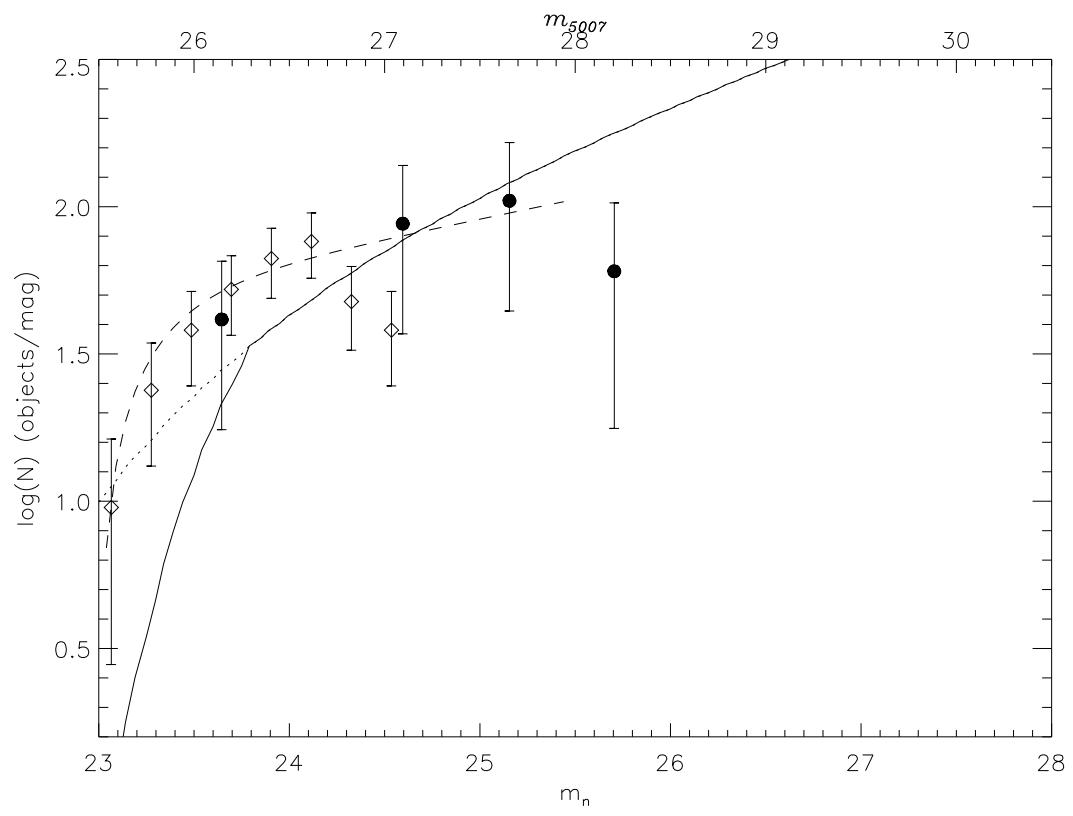

Fig. 8. - Luminosity function of the ICPNe sample selected in the RCN1 field. The dashed line shows the PNLF for a distance modulus of 30.29, convolved with the photometric errors. On the same plot the continuos line shows the expected LF in our narrow filter for the Ly $\alpha$ population in the field at redshift $\mathrm{z}=3.13$ from Steidel et al. (2000), see discussion in the text. The faint dotted line shows the expected contribution from the Ly $\alpha$ emitters with V magnitudes brigther than 24.75. Full dots indicate the LF in our narrow band filter from Ly $\alpha$ emitters in the blank-field survey by Cowie \& $\mathrm{Hu}$ (1998). 
Jacoby, G.H. 1989, ApJ, 339, 39

Jacoby, G., Ciardullo, R, Ford, H. 1990, ApJ, 356, 332

McMillan, R., Ciardullo, R., Jacoby, G.H. 1993, ApJ, 416, 62

Melnick, J., White, S.D.M., Hoessel, J. 1977, MNRAS, 180, 207

Méndez, R.H., Kudritzki, R.P., Ciardullo, R., Jacoby, G.H. 1993, A\&A , 275, 534

Méndez, R.H. et al. 1997, ApJ, 491, L23

Méndez, R.H., Riffeser, A., Kudritzki, R.P. et al. 2001, ApJ, in press

Neilsen, E.H., Tsvetanov, Z.I. 2000, ApJ, 536, 255

Oke, J.B. 1990, AJ, 99, 1621

Pierce, M., Tully, R.B. 1988, ApJ, 330, 579

Renzini, A., Buzzoni, A. 1986, in Spectral Evolution of Galaxies, ed. C. Chiosi and A. Renzini (Dordrecht:Reidel), 195

Smith, H.A. 1981, AJ 86, 998

Steidel, C.C., Adelberger, K.L., Giavalisco, M., Dickinson, M., Pettini, M. 1999, ApJ, 519, 1

Steidel, C.C., Adelberger, K.L., Shapley, A.E., Pettini, M., Dickinson, M., Giavalisco, M. 2000, ApJ, 532, 170

Teplitz, H.I. et al. 2000, ApJ, 542, 18

Theuns, T., Warren, S. 1997, MNRAS, 284, L11

Thuan, T.X., Kormendy, J. 1977, PASP, 89, 466

Tonry, J.L., Ajhar, E.A., Luppino, G.A. 1990, AJ, 100, 1416

Uson, J.M., Boughn, S.P., Kuhn, J.R. 1991, ApJ, 369, 46

Vilchez-Gómez, R. Pelló, R., Sanahujia, B. 1994, A\&A, 283, 37

Welch, G.A., Sastry, G.N. 1971, ApJ, 169, 13

West, M.J., Blakeslee, J.P. 2000, ApJ, 543, L27

Yasuda, N., Fukugita, M., Okamura, S. 1997, ApJSS, 108, 417

Zwicky, F. 1951, PASP, 63, 61 
Table 1. Number of modelled point-like objects and spurious detections recovered from simulations.

\begin{tabular}{ccc}
\hline \hline LOWTHRESHOLD & Point-like objects & Spurious detections \\
\hline $0.7 \sigma$ & 680 & 1001 \\
$0.8 \sigma$ & 567 & 237 \\
$0.9 \sigma$ & 478 & 66 \\
$1.0 \sigma$ & 410 & 30 \\
$1.1 \sigma$ & 362 & 16 \\
$1.2 \sigma$ & 310 & 11 \\
\hline
\end{tabular}

